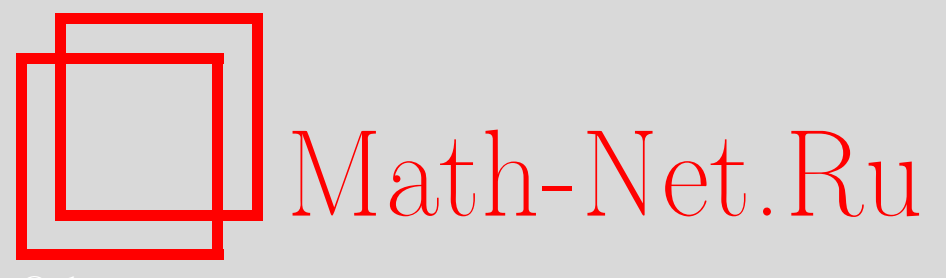

В. Соловьев, С. Дворянинов, Что такое фазовый портрет, Квант, 2019, номер 12, 27-30

DOI: https://doi.org/10.4213/kvant20191205

Использование Общероссийского математического портала Math-Net.Ru подразумевает, что вы прочитали и согласны с пользовательским соглашением http://www . mathnet.ru/rus/agreement

Параметры загрузки:

IP: 54.197.217.227

26 апреля 2023 г., 09:05:00

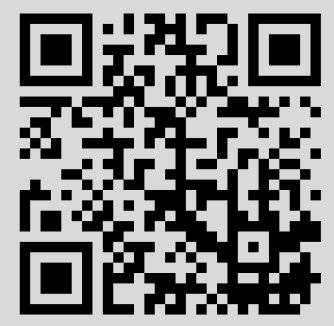




\section{Что такое фазовый портрет}

\section{В.СОЛОВЬЕВ, С.ДВОРЯНИНОВ}

Следовать за мыслями великого человека есть наука самая занимательная.

А.С.Пушкин

\section{Космология Фридмана}

О том, что Вселенная расширяется, в наши дни многие наверняка слышали, читали и знают. А какой может быть эволюция наблюдаемой Вселенной в будущем? Удивительно, но еще в 1922 году А.А.Фридман в статье «О кривизне пространства» определил возможные варианты развития Вселенной. Сейчас мы хотим познакомить читателей с этой статьей. Конечно, рассказать о работе ученого непросто. Свою статью Фридман адресовал коллегам физикам, университетским профессорам. Тем не менее, мы постараемся сделать доступной основную канву мыслей ученого, внесшего решающий вклад в понимание структуры космоса.

Начнем с простой школьной задачи. На плоскости с координатами $\left(x_{1} ; x_{2}\right)$ надо изоб-

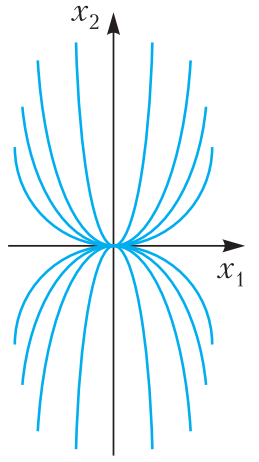

РИс. 1 разить множество точек, координаты которых удовлетворяют уравнению $x_{2}=c x_{1}^{2}$, где $c-$ произвольная постоянная. Такое уравнение задает семейство квадратичных парабол (рис.1). Эволюция этих парабол такова: при возрастании параметра $c$ от $-\infty$ до нуля ветви парабол отходят от отрицательной полуоси ординат и приближаются к оси абс-

Окончание. Начало - в предыдущем номере журнала.

DOI: https://doi.org/10.4213/kvant20191205 цисс. При $c=0$ имеем прямую линию. При изменении $c$ от нуля до $+\infty$ ветви парабол неограниченно приближаются к оси ординат.

Ясное дело, что координаты точек плоскости и параметр можно обозначать разными буквами. На плоскости с координатами $\left(R ; R^{\prime}\right)$ уравнение $R^{2}+\lambda R^{\prime 2}=1$ при каждом отрицательном значении параметра $\lambda$ задает две ветви гиперболы (рис.2). При $\lambda=0$

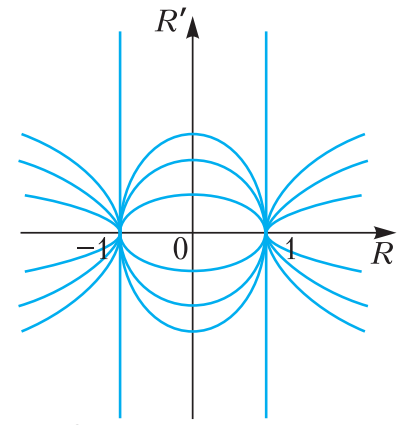

Pис. 2

получаем две прямые $R= \pm 1$. При положительных значениях $\lambda$ получаем эллипсы, которые при возрастании параметра от нуля до $+\infty$ отходят от этих прямых и неограниченно приближаются к отрезку $-1 \leq R \leq 1$ оси абсцисс.

Мы специально использовали непривычные для школы буквы $R, R^{\prime}$ и $\lambda$ для обозначения координат кривой второго порядка и для параметра. Почему? Да потому, что именно такие обозначения мы видим в одном знаменитом уравнении!

Уравнение Фридмана. В 1922 году работавший в Петрограде Александр Александрович Фридман впервые получил и исследовал такое уравнение:

$$
\frac{3 R^{\prime 2}}{R^{2}}+\frac{3 c^{2}}{R^{2}}-\lambda=\frac{\kappa}{2} c^{2} \rho .
$$

Об этом уравнении можно прочитать, например, в статье С.Дворянинова и В.Соловьева «Космология Фридмана: горы реальные и потенциальные» («Квант», 2017, №1, 2). Здесь $c$ и к - постоянные: $c$ - скорость света, $\kappa=\frac{16 \pi G}{c^{2}}$, где $G-$ гравитационная постоянная из закона всемирного тяготения Ньютона; $\rho=\frac{M}{2 \pi^{2} R^{3}}$, где $M-$ постоянная положительная величина. 
Сейчас мы будем рассматривать уравнение (*) как алгебрачческое уравнение на плоскости $\left(R ; R^{\prime}\right)$ с фиксированным положительным параметром $\lambda$, причем $R>0$. Наша ближайшая цель сугубо математическая показать на плоскости точки, координаты которых удовлетворяют этому уравнению. Для этого запишем его в виде

$$
R^{\prime 2}=-c^{2}+\frac{\lambda}{3} R^{2}+\frac{a}{3 R},
$$

где новая постоянная $a$ выражается через старые постоянные. Это уравнение связывает координаты $R$ и $R^{\prime}$.

Для построения графика этой функции на плоскости $\left(R ; R^{\prime}\right)$ рассмотрим при положительных значениях аргумента $R$ вспомогательную функцию

$$
y(R)=-c^{2}+\frac{\lambda}{3} R^{2}+\frac{a}{3 R} .
$$

При малых $R$ график этой функции ведет себя как гипербола, имеющая асимптотой ось ординат, а при больших $R$ - как парабола. Эта функция имеет единственную точку минимума (проверьте!). Минимальное значение в зависимости от $\lambda$ может быть положительным, равным нулю и отрицательным (рис.3). Если значения $\lambda$ достаточно велики,

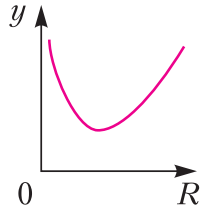

Pис. 3
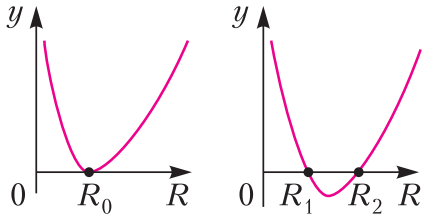

то минимальное значение оказывается положительным, и тогда $y(R)>0$ при всех положительных значениях $R$. При других значениях $\lambda$ эта функция может иметь одно нулевое значение - в точке $R_{0}$ или два - в точках $R_{1}$ и $R_{2}$. При неотрицательных значениях $y$ «извлекаем квадратный корень» и приходим к графику уравнения Фридмана (рис.4).

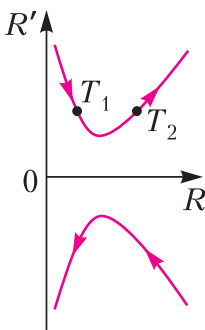

Pис. 4

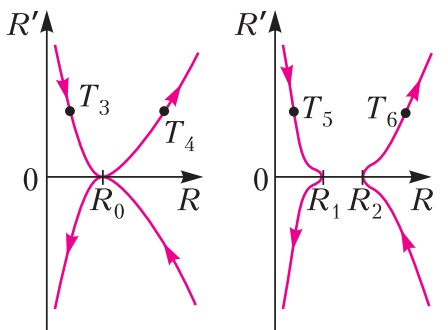

Фазовые траектории. Теперь пришло время сказать, что в уравнении Фридмана величина $R$ зависит от времени: $R=R(t)$ и что $R^{\prime}$ есть производная $R^{\prime}(t)$ этой величины. Стало быть, уравнение $(*)$ - это дифференииальное уравнение, а кривые на рисунке 4 - это фазовые траектории (о них рассказывается в первой части статьи). Стрелки вдоль траекторий показывают направление движения фазовой точки в зависимости от времени. В верхней полуплоскости $R^{\prime}(t)>0$, поэтому функция $R(t)$ здесь возрастает и вдоль каждой фазовой траектории фазовая точка движется вправо. В нижней полуплоскости - наоборот, фазовая точка движется справа налево. Этот рисунок не является фазовым портретом уравнения (*), на нем показано лишь несколько фазовых траекторий, так как в этом уравнении нет произвольной постоянной.

В зависимости от $\lambda$ на фазовой плоскости $\left(R ; R^{\prime}\right)$ есть или одна точка покоя - $\left(R_{0} ; 0\right)$, или две $-\left(R_{1} ; 0\right)$ и $\left(R_{2} ; 0\right)$. Единственной точки покоя другие фазовые траектории не достигают никогда, а вот в каждую из двух точек покоя фазовая траектория попадает за конечное время (в этих двух точках касательная к траектории вертикальна).

Заметим, что фазовые траектории вблизи точек покоя ведут себя подобно графикам двух дифференциальных уравнений $x^{\prime 2}=x^{2}$ и $x^{\prime 2}=-4 x$. Решения первого уравнения $x(t)=c e^{ \pm t}$ с ненулевым начальным условием никогда в ноль не обращаются, т.е. точка покоя для них недостижима. Второе уравнение имеет, например, решение $x(t)=-(t-1)^{2} \quad$ с начальным условием $x(0)=-1$, оно обращается в ноль при $t=1$, т.е. за конечное время. Соответствующая траектория попадает в точку покоя за конечное время, может оставаться там при $1 \leq t \leq t_{1}$, а затем ее покинуть и двигаться по закону $x(t)=-\left(t-t_{1}\right)^{2}$. Такую точку покоя можно назвать точкой поворота (возврата). О связи этого свойства фазовых траекторий с практическими задачами причаливания кораблей рассказано в статье С.Дворянинова, З.Краутера и В.Протасова «Сколько времени длится причаливание» («Квант», 2017, №11).

А каков же физический смысл величины $R$ ? 
Как устроена видимая нами Вселенная.

Для муравья, ползущего по веревке, весь мир сводится к двум направлениям: вперед или назад. Это - одномерный мир. Для ребенка, еще не вставшего на ноги и играющего на полу, мир не просто плоский, двумерный, он вдобавок еще и ограничен размером его детской. Таков же мир животных, обитающих на заповедном острове и не умеющих плавать.

Долгое время человек представлял поверхность Земли плоской. Ощущения и непосредственные наблюдения могут формировать ложные представления. Если человеку завязать глаза и попросить его двигаться по прямой линии, то его траектория окажется похожей на окружность. У большинства людей шаг правой ногой чуть-чуть длиннее шага левой, и потому на каждом шаге происходит малый, но все же поворот. Идти по прямой не получается. При этом человек убежден, что он идет строго по прямой. Вообразим еще, что человек может обозреть на поверхности Земли лишь круг радиусом, скажем, 10 метров. Поставим перед ним задачу продолжить нарисованный на земле прямолинейный отрезок длиной 5 метров. Пусть вся поверхность Земли - суша. Человек, продолжая этот отрезок с помощью, например, метровой линейки, несомненно удивится, вернувшись в исходную точку с противоположной стороны.

А как устроен видимый нами мир? Если на ракете лететь все время, как нам кажется, по прямой, то где в конце концов мы окажемся? Эйнштейн в своей первой статье по космологии в 1917 году предположил, что наш мир искривлен и представляет собой трехмерную сферу. В свое время люди считали наш мир куском плоскости, на деле Земля оказалась двумерной сферой. Давайте по аналогии вслед за Эйнштейном примем допущение о том, что и наш мир искривлен и это - сфера, но трехмерная. Она - «поверхность» четырехмерного шара радиусом $R$, и, как показали математики, ее объем равен $V=2 \pi^{2} R^{3}$. При таком подходе $R$ называют радиусом мира, а величину $\rho=\frac{M}{V}-$ средней плотностью вещества во Вселенной, где $M$ - масса вещества.

У равнения Фридмана есть следствия уравнений общей теории относительности (ОТО) и сделанных Эйнштейном предположений об однородности и изотропности пространства. Десять уравнений ОТО сводятся к двум дифференциальным уравнениям, наиболее простое из которых - уравнение $(*)$ и называется сейчас уравнением Фридмана. Это уравнение оставляет Вселенной только одну степень свободы - ее радиус.

При выводе своих уравнений Фридман считал, вслед за Эйнштейном, что Вселенная в среднем равномерно заполнена пылью, которая не создает никакого давления. Пространство однородно и изотропно, т.е. его свойства одинаковы по всем направлениям и в любой точке.

Величина $\lambda$, входящая в уравнение $(*),-$ это введенная Эйнштейном космологическая постоянная. Эйнштейн ввел ее для того, чтобы его уравнения имели пространственно однородное статическое решение, соответствующее точке покоя $R_{0}$, для которого $R$ есть постоянная величина, не зависящая от времени.

В наши дни мы наблюдаем расширяющуюся Вселенную. Об этом свидетельствует так называемое красное смещение света от далеких галактик. Следовательно, в настоящий момент фазовая точка, соответствующая наблюдаемому состоянию Вселенной, - это какая-то из точек $T_{1}-T_{6}$ (см. рис.4) первой координатной четверти, в этой четверти производная $R^{\prime}(t)>0$ и $R(t)$ возрастает. Фазовая траектория, проходящая через такую точку, определяет будущее Вселенной, ее эволюцию. Здесь возможны несколько сценариев. Напомним, что данные наблюдений (а именно, реликтовое излучение, открытое в 1965 году) говорят, что в самом начале эволюции Вселенной произошел «Большой взрыв». Тогда радиус $R$ практически равнялся нулю, и поэтому нынешнему состоянию Вселенной не могут соответствовать точки, обозначенные как $T_{4}$ и $T_{6}$. Для таких точек в прошлом $R \neq 0$.

Первый сиенарий. С ходом времени Вселенная продолжит расширение. При этом скорость расширения либо будет сначала убывать (для точки $T_{1}$ ), достигнет положительного минимума и затем начнет неограниченно возрастать, либо будет монотонно и неограниченно возрастать (для точки $T_{2}$ ). В обоих случаях Вселенная будет монотонно и неограниченно расширяться. 
Второй сценарий. Скорость расширения будет монотонно убывать (для точки $T_{3}$ ), неограниченно приближаясь к нулю. Вселенная будет расширяться, но при этом величина $R$ останется ограниченной. Этот процесс будет длиться бесконечно долго. Время приближения к точке $\left(R_{0} ; 0\right)$ бесконечно. Здесь фазовые траектории не пересекаются, подобно тому, как сепаратриса на фазовом портрете маятника не содержит точку покоя (см. первую часть статьи).

третий вариант. Для точки $T_{5}$ Вселенная будет расширяться, скорость расширения будет уменьшаться и через некоторое конечное время станет равной нулю. Вселенная окажется в стационарном состоянии с радиусом $R=R_{1}$ и может оставаться таковой сколь угодно долго. А может затем и начать сжиматься с неограниченно возрастающей скоростью, при этом радиус Вселенной будет стремиться к нулю. Это объясняется тем, что в точке $\left(R_{1} ; 0\right)$ нарушается единственность решения дифференциального уравнения $(*)$ и фазовые траектории пересекаются.

Реальная история. Фридман умер в 1925 году, так и не узнав о том, что Вселенная и впрямь подчиняется его уравнению, что она расширяется (1929 г.), что имел место Большой взрыв (1965 г.) и что расширение в нашу эпоху происходит с ускорением (1998 г.). Наш анализ мог бы быть актуальным в период между 1965 и 1998 годами. Сейчас из всех сценариев лишь точка $T_{2}$ на качественном уровне соответствует имеющимся данным. Количественную связь между двумя параметрами $a$ и $\lambda$, следующую из современных данных, мы обсуждали в упомянутой статье «Космология Фридмана...».

\section{Н АШ И НАБЛЮДЕНИЯ}

\section{КУДА ДУЕТ ВЕТЕР?}

Осенью или зимой в тихую и туманную погоду ветки на деревьях и кустах, провода, мачты антенн покрываются мохнатым инеем или инееобразным ледяным слоем - изморозью. Иней и изморозь состоят из мельчайших частичек льда, слипшихся в виде столбиков, тонких перьев, игл и т.п. Обычно инеем называют снежные кристаллы, которые образуются из водяных паров в насыщенной влагой атмосфере, а изморозью - ледяной осадок, возникший из переохлажденных капелек тумана. Иней и изморозь могут появляться, а могут и исчезать - если вдруг потеплеет или подует сухой ветер (кристаллики льда тают или испаряются соответственно).

Оказывается, даже небольшой ветерок способен сильно изменить картину морозных узоров. Посмотрите на фотографию ветки растения, покрытого изморозью. Ветка была сфо-

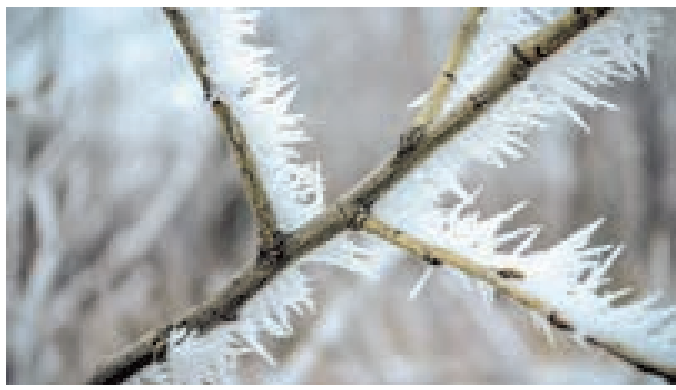

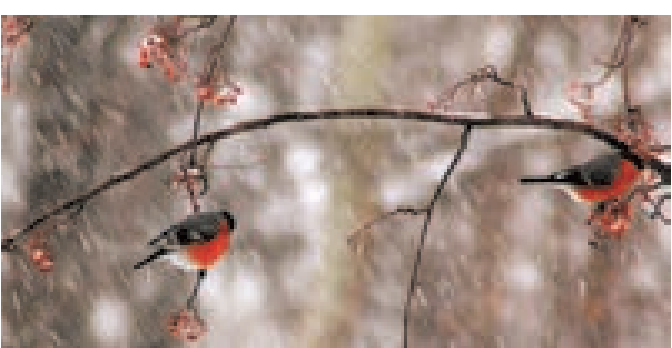

тографирована морозным утром, когда был очень сильный туман, гололед и дул слабый ветер. Можно ли по этой фотографии однозначно установить направление ветра? Давайте попробуем.

Так как изморозь образуется из капелек тумана, она появляется на предметах преимущественно с подветренной стороны и может быстро расти при ветре. В нашем случае во время съемки погодные условия были благоприятны росту изморози (а не таянию льда), а слабый ветер способствовал появлению анизотропии формы ледяных наростов. Поэтому, глядя на фотографию, легко определить преимущественное направление ветра - слева направо.

А теперь подумайте, как по виду ледяных кристалликов изморози или инея (не привлекая каких-либо метеоданных) узнать, давно или недавно образовался ледяной нарост на ветках. 